\title{
Design and Implementation of Automatic Landing Control System for UAV Based on GPS
}

\author{
Jia-xian Li \\ \{ lijiaxianln@163.com\} \\ School of Mechanical and Electronic EngineeringEastern Liaoning University, Liaoning, Dandong, \\ 118003 , China
}

\begin{abstract}
UAV's development history and UAV variety of launch and recovery are introduced in this paper, study UAV two automatic landing system and the advantages and disadvantages of the two compared and according to the existing conditions determined a suitable landing system scheme is adopted. In the determined on the basis of the program established UAV taxiing nonlinear model and landing model, respectively for automatic takeoff and landing control system design. The elevator control and nose wheel steering control are designed respectively for UAV Ground taxiing longitudinal and lateral control law using glide beam guiding system and auto leveling control system and lateral beam guiding system, combining the control law are designed UAV longitudinal automatic landing system control laws and lateral automatic landing control law and in the design of the control system and several common wind disturbance, adopted the surname simulation to verify the robustness of the control system. The design of UAV automatic take-off and landing control system program, basically meet the requirements, I believe the work of this paper will provide a useful reference for the future development of the UAV automatic take-off and landing technology.
\end{abstract}

Keywords: Unmanned aerial vehicle; automatic take-off and landing; control; instrument landing system

\section{Introduction}

Unmanned aerial vehicle is a kind of unmanned, reusable aircraft, which was born in the century, after the era of greater development, in the military and civil fields have been widely used. At first the UAV is used as a test target. Later in some countries they have developed a unmanned reconnaissance aircraft, unmanned aircraft, unmanned helicopter. Makes people realize the importance of unmanned aerial vehicle (UAV) military value is the Middle East War, war in the Middle East Small UAV emerge, the Gulf War in the use of a large small unmanned aerial vehicle (UAV), and in the Kosovo war in medium altitude long endurance without man-machine burgeoning, in the middle of the Afghanistan and Iraq war is played a powerful, during the war in Afghanistan, "Predator" first launch missiles, is also the first time UAV missile launch, to attack the ground, put in the Iraq war to the United States for UAV is most of the time, the number is three times the war in Afghanistan. In the military, no machine can be as airborne reconnaissance platforms and weapons platform [1], by carrying different devices, executive, surveillance and reconnaissance, ground attack, electronic jamming, communications relay, target location, attack damage effectiveness evaluation tasks in civilian 
areas, no machine can be used in field monitoring, meteorological observation, highway patrol, exploration and mapping, flood monitoring, power line patrol, forest fire prevention and rescue. It can be seen that the UAV has broad application prospects in the military and civil fields. The development of the UAV has mainly experienced three stages: the first stage is the five in the last century, sixty years, formed a development period of an unmanned drone, which is mainly used for the performance of the new developed aircraft and missile test and identification of the second stage is the birth of unmanned reconnaissance aircraft, mainly used for reconnaissance and battlefield on the battlefield the assessment of the third stage is the last century ninety's UAV rapid development stage, in this period of UAV has been widely used in the battlefield, especially small UAV emerge, war in the Middle East, the development of UAV series at the same time, there are three hotspots, long endurance UAV unmanned the fighter and mini uav. In this period the United States "Global Hawk" has also developed, and each country has set up a professional uav.

\section{System Scheme Design}

The development of instrument landing system is relatively mature, but there can only provide a single and fixed slide, beam covered area is small, multipath interference and other serious shortcomings of microwave landing system mainly has the advantages of high guide accuracy, the proportion of large coverage area, can provide a variety of approach route and all-weather guidance function, but the cost is high, and the ground airborne equipment requirement is high, with a larger price, has limited the development of the satellite navigation system is developed following the U.S. military, inertial navigation, another major development of navigation technology, with a global, all-weather positioning capabilities, protection signal of high positioning accuracy, wide application range and relatively low cost advantages, but there are also due to a country of human interference control, large error and poor reliability of wartime domestic big dipper system is still in the stage of perfect reason cannot be applied in this design. There are many similarities between the UAV's takeoff and landing system and manned aircraft, but it is more different from the characteristics of the automatic takeoff and landing of the UAV [2].

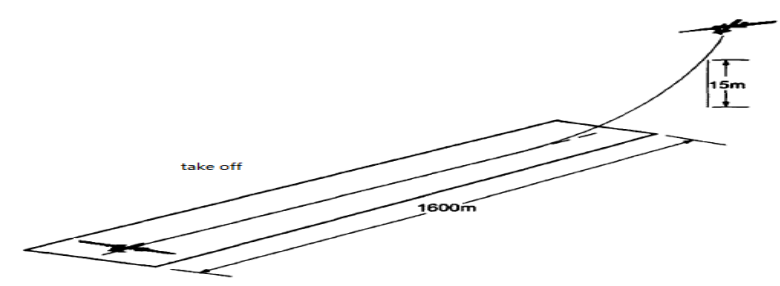

Fig. 1. UAV take-off sketch map

\subsection{Automatic Landing Scheme Design}

To achieve the level of performance of the system is strictly an automatic approach rather than an automatic landing. The automatic landing of the automatic flight control system has the corresponding reliability and control accuracy, and it is required to be used for landing control of the ground and the aircraft navigation equipment to achieve the corresponding level. With the development of digital control and redundancy technology, the automatic flight 
control system is no more than the automatic flight control system. Modern large civil aircraft have been put into. Level or level of automatic landing capability as a basis for the requirements of the aircraft and equipment. And at present the world can achieve the level of landing requirements of the international airport runway has been up to more than 100. Allweather automatic landing technology development has not suspended, it is accompanied by the development of navigation equipment and forward, and with the application of satellite technology for the implementation of the characteristics of communication, navigation, surveillance and air traffic management global navigation system into a new stage of development. The instrument landing system was first adopted by the International Civil Aviation Organization in the year of the international civil aviation organization, and as a standard approach and landing equipment. Since then the system has been greatly improved. In fact, the reliability and the improvement of the instrument landing system have been achieved. However, an instrument landing system on any of a class of landing can provide the service ability, in a certain extent depending on external factors, such as stations in the vicinity of buildings, trees, undulating terrain and low flying aircraft and other interference effects, the emission beam generated strong noise level or beam bending. And this system can only provide a single and fixed approach route, from the fundamental limits, such as the curve into the near, subsection into the near and large decline angle into the near and other flexible approach to the use of [3].

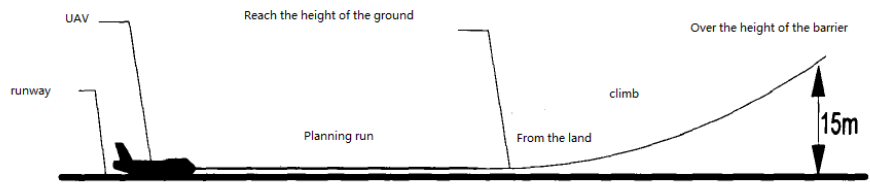

Fig. 2. System takeoff trajectory

\subsection{Overall Landing Design}

The overall program is mainly based on the principle and equipment of the automatic landing aircraft landing program, on this basis to improve, so that the program is more suitable for the automatic landing of unmanned aerial vehicles. For someone to fly the aircraft, especially the aircraft automatic landing ground equipment on the ground is more complicated, but also requires more capital investment. The more important is that the complex system is not suitable for the automatic landing of uav. So the scheme is a modification scheme is proposed based on a manned aircraft automatic landing techniques. The automatic landing system is an automatic landing and landing position sensor platform based on the instrument landing system. No UAV automatic take-off and landing position sensor includes a beacon positioning transceiver system, directional beacon transceiver system, laser sensor height, airspeed sensor and the lifting speed sensor. The scheme is based on the "internal baseline" method. Due to the high brightness, high accuracy and high color of laser, the high precision and wide range of technology in the field of technology is far more than that of the optical and infrared. The absolute precision of laser ranging can reach the order of $1.1 \mathrm{~mm}$. From the simulation results, we can see, when the landing process without man-machine lateral deviation, automatic control system can rapid control of the UAV to correct landing route, the system response during maximum inclination angle, maximum the sideslip angle and yaw angle control in the basic requirements within the scope of. Similarly, when the initial lateral deviation is increased, the UAV can quickly return to the route, but the 
range of changes in each control is slightly larger. Lateral beam automatic control system technology is relatively mature, and in front of the automatic landing control system design using the instrument landing system glide beam guidance system. Therefore, lateral automatic control system still uses the instrument landing system of the lateral beam guiding system [4].

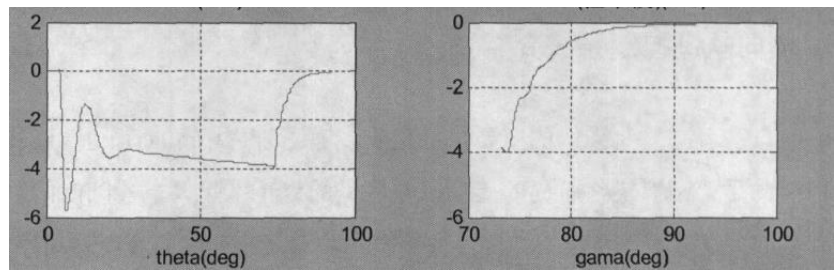

Fig. 3. System simulation results chart

\section{System Design and Implementation}

UAV's takeoff and landing phases of flight characteristics different to other phases of flight flight characteristics, thus the UAV automatic take-off and landing control system design is also different to the other phases of flight control system design, the general design of flight control system is based on small disturbance linearization method, namely in the equilibrium point of reference motion of nonlinear equations of motion of the plane of the small disturbance linearization, a linearized model of the aircraft, then the control system design. And take off stage of aircraft is an acceleration stage, flight speed is relatively low, there is no a for linearized equilibrium state and thus does not proceed small disturbance linearization of the intermediate stage, using a nonlinear control design method is more suitable for the actual situation. In the concrete design process, we divide the motion of the UAV into two parts: longitudinal and lateral. Due to the present stage without UAV related data and reference other automatic take-off and landing control system technical requirements, we refer to the domestic similar transport data, using the transport related data as the UAV's takeoff and landing state data. At this stage are unable to obtain wig data and landing gear data and in order to simplify the calculation, the kinematic and dynamic analysis of the aircraft's ground motion, we ignore the elasticity of the tire, the tires and wheels in the same plane and according to the existing data assumed landing gear and engine related data for subsequent calculations. Because at this stage to aircraft data is very limited, according to the aircraft data has been obtained to determine longitudinal and lateral skating motion equation of gas dynamic derivative and other parameters value calculation formula. In the takeoff stage, according to the thrust curve can determine the taxiing of UAV available thrust range. In order to determine the taxiing of the thrust value. The resistance is obtained by one dimensional linear interpolation of the data sheet [5].

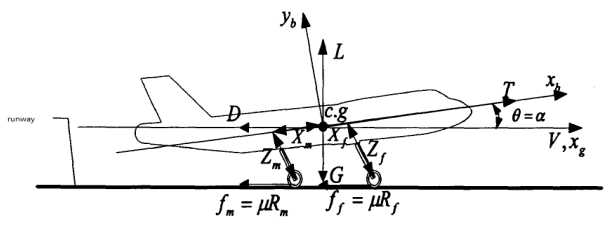


Fig. 4. Force sketch map of UAV

\section{Conclusion}

Takeoff and landing is a complex phase of flight, is a key technology of development of human, today without the requirement of development of computer technology, this paper first introduced the UAV's background and development history, and at home and abroad UAV launch and recovery. Then the automatic take-off and landing system of the two sets of UAV is studied and compared, and a suitable scheme is selected according to the requirements of the landing and the actual conditions. Then in has been obtained from the aircraft data based on established UAV taxiing nonlinear model and landing nonlinear model, using design methods are used to design the UAV automatic takeoff control law and the automatic landing control law. Based on the designed control rate, the design and simulation are carried out in the MATLAB environment. The results show that the designed control system has good robustness.

\section{Acknowledgements}

Project supported by Liaoning Science and Technology Department, [2012]261.

\section{References}

[1] Zhang Libin, Su sciencsys. Analysis on aircraft crosswind landing problem. Flight mechanics. No. 04. (2012)

[2] Xu Tingxue, Cao Yunfeng, Luo Feng. Research on automatic landing system of an unmanned aerial vehicle. Journal of Nanjing University of Aeronautics \& Astronautics. No. 05. (2014)

[3] Ding Yongfei. Research on the application of the integrated navigation and landing system of UAV with DGPS / autopilot / altimeter. avionics technology. No. 03. (2013)

[4] Xie Shifu. Review of the development of navigation technology and system. electronic quality. No. 01. (2012)

[5] Zhang Zhisheng, Chen Huaimin, Wu Chengfu, Ma Songhui. A new type of small UAV flight control strategy for the study of. Flight dynamics. No. 04. (2013) 